\section{Dosage compensation for the birds}

JA Birchler February 2009

S exromosomes evolve from a homologous pair of chromosomes to become cytologically distinct. Consequently, fertilization produces one of two different chromosomal combinations that will initiate either the male or female developmental program. One sex will be heterogametic (have one of each type of sex chromosome) and the other sex will be homogametic (have a pair of one type). In the process of this evolution, one of the pair of chromosomes can become quite degenerative, leaving its former homolog as the sole bearer of many genes. This situation raises the issue of whether the heterogametic sex has reduced expression of these genes, which might be detrimental, or whether a process called dosage compensation sustains their expression. In humans, males are the heterogametic sex, having both $X$ and $Y$ chromosomes, whereas females have two Xs. However, this state of affairs is not found universally in all species. For example, in birds and butterflies females are heterogametic-with Z and W chromosomes; males have two $\mathrm{Z}$ chromosomes.

Recent studies by Mank and Ellegren (2008) have used global gene profiling of male and female chickens, in different tissues and developmental stages, to shed light on the unresolved issue of dosage compensation in birds. They have established that compensation occurs on a gene-by-gene basis and that compensation can come and go for individual genes during development. Similar results have also been reported by Itoh et al. (2007). This distinctive mode of dosage compensation has intriguing implications for the evolution of dosage compensation mechanisms in general.

The $Z$ chromosome of chickens contains more than 700 known genes, whereas the $W$ chromosome is quite depauperate. Mank and Ellegren examined 630 Z-linked genes in male and female brains, the least sex-biased tissue, at four time points in development. They compared the expression with that in the gonads, the most sex-biased tissue, at three time points. When the average gene expression is calculated for the $Z$ chromosome, its mean is indeed greater in males with two $\mathrm{Z}$ chromosomes than in females with only one, but does not approach the two-fold level that would indicate the complete lack of dosage compensation. The ratio differs between both the two tissues examined and the developmental time points, with an indication that greater compensation occurs during the embryonic stages than in adults. The ratios vary gene-by-gene over the length of the $\mathrm{Z}$ chromosome. This piecemeal pattern of compensation differs from the three most thoroughly studied examples of sex chromosome dosage compensation-flies, worms and mammals (Birchler et al., 2006) -in which there is a greater percentage of X-linked genes that are compensated, in these cases in males.

The dosage compensation in birds is, however, somewhat similar to that which occurs in aneuploids (individuals with either fewer or more chromosomes than the normal balanced diploid) of non-sex chromosomes. This intrinsic type of dosage compensation has been documented in maize (Birchler, 1979), flies (Devlin et al., 1982) and humans (Altug-Teber et al., 2007). It operates on a gene-by-gene basis, and exhibits developmental specificity (Guo and Birchler, 1994). This similarity raises the possibility that the chicken-sex chromosome dosage compensation represents a tweaking of intrinsic compensation. Intrinsic compensation might occur as a consequence of the stoichiometric imbalance in gene-regulatory processes, (Birchler et al., 2001; Veitia et al., 2008) causing an inverse dosage response. However, there are distinctions between the Z-chromosome compensation and the intrinsic compensation observed in aneuploids in other species. First, aneuploidy of chromosomes as large as the $\mathrm{Z}$ chromosome results in lethality or highly abnormal phenotypes (Birchler et al., 2001), so one might expect that selection has occurred to compensate the Z-linked genes that are the most detrimental when dosage is altered. Second, aneuploids often show some level of trans-acting effects that modulate genes on other chromosomes, potentially because of the dosage effects of regulatory genes that are not compensated (Birchler et al., 2001; Veitia et al., 2008). Female chickens have only one $\mathrm{Z}$ chromosome, and are obviously viable. In the experiments, there was no evidence for significant autosomal modulations so, if there is a relationship between intrinsic dosage compensation mechanisms and that of the $\mathrm{Z}$ chromosome, then either regulatory genes are particularly prone to selection for compensation, for which Mank and Ellegren note there is some rudimentary evidence, or a mechanism has evolved to prevent any dosage effects of the $Z$ chromosome on autosomal expression.

Does the compensation in birds represent a primordial form that is common to other types of sex chromosome dosage compensation? Certainly, the divergence of the $\mathrm{Z}$ and $\mathrm{W}$ chromosomes is not recent, so one should not consider the avian mode of dosage compensation as an 'early' step in compensation evolution. However, because of the similarities to intrinsic compensation noted above, avian compensation might present an example that is instructive for understanding the evolution of other forms of compensation in flies, worms and mammals. Recent studies have indicated a common theme in these three taxa. In each case, there is a two-fold upregulation of the single $X$ chromosome despite the differences in sex-specific chromatin modifications that are unique to each species (Gupta et al., 2006; Nguyen and Disteche, 2006; Birchler et al., 2006). Indeed, triple $X$ metafemales in flies show dosage compensation that requires a downregulation to achieve expression similar to that in normal females (Birchler et al., 1989) and there are no known chromatin differences in this sex chromosome between aneuploid and diploid females. Also, in mammals, tissues without an X:autosome imbalance, such as haploid cells in the gonads, show no upregulation of the $\mathrm{X}$ chromosome (Nguyen and Disteche, 2006). The upregulation of the single active $X$ in mammals is independent of the process of $X$ inactivation. So, it is possible that all cases of dosage compensation modify the intrinsic stoichiometrically based mechanism to some degree, for equalization of the expression of crucial sex-linked genes between the sexes and with the remainder of the genome. Different cases of sex chromosome evolution might have solved the issues of critical gene compensation and trans-acting effects on the autosomes in different ways. The new results on chicken dosage compensation add new data for understanding the similarities 
and differences among sex-chromosomal dosage compensation mechanisms. Dr JA Birchler is at the Division of Biological Sciences, University of Missouri, Columbia, MO, USA.

e-mail: BirchlerJ@Missouri.edu

Altug-Teber O, Bonin M, Walter M, Mau-Holzman UA, Dufke A, Stappert H et al. (2007). Specific transcriptional changes in human fetuses with autosomal trisomies. Cytogenet Genome Res 119: 171-184.

Birchler JA (1979). A study of enzyme activities in a dosage series of the long arm of chromosome one in maize. Genetics 92: 12111-11229.

Birchler JA, Bhadra U, Pal Bhadra M, Auger DL (2001). Dosage dependent gene regulation in higher eukaryotes: implications for dosage compensation, aneuploid syndromes and quantitative traits. Dev Biol 234: 275-288.

Birchler JA, Fernandez H, Kavi H (2006). Commonalities in compensation. Bioessays 28: 565-568.
Birchler JA, Hiebert JC, Krietzman M (1989). Gene expression in adult metafemales of Drosophila melanogaster. Genetics 122: 869-879.

Devlin RH, Holm DG, Grigliatti TA (1982). Autosomal dosage compensation in Drosophila melanogaster strains trisomic for the left arm of chromosome 2. Proc Natl Acad Sci USA 79: 1200-1204.

Guo M, Birchler JA (1994). Trans-acting dosage effects on the expression of model gene systems in maize aneupoids. Science 266: 1999-2002.

Gupta V, Parisi M, Sturgill D, Nuttall R, Doctolero M, Dudko OK et al. (2006). Global analysis of X-chromosome dosage compensation. J Biol 5: 3.

Itoh Y, Melamed E, Yang X, Ka P, Wang S, Yehya N et al. (2007). Dosage compensation is less effective in birds than in mammals. J Biol 6: 2 .

Mank JE, Ellegren H (2008). All dosage compensation is local: gene-by-gene regulation of sex-biased gene expression in the chicken $Z$ chromosome. Heredity.

Nguyen DK, Disteche CM (2006). Dosage compensation of the active $X$ chromosome in mammals. Nat Genet 38: 47-53.
Veitia R, Bottani S, Birchler JA (2008). Cellular reactions to gene dosage imbalance: genomic, transcriptomic and proteomic effects. Trends Genet 24: 390-397.

\section{Editor's suggested reading}

Fairbairn DJ, Roff DA (2006). The quantitative genetics of sexual dimorphism: assessing the importance of sex-linkage. Heredity 97: 319-328.

Johnson RM, Shrimpton JM, Cho GK, Heath DD (2007). Dosage effects on heritability and maternal effects in diploid and triploid Chinook salmon (Oncorhynchus tshawytscha). Heredity 98: 303-310.

Marais GA (2007). The Hill-Robertson effects extend from nucleus to mitochondria. Heredity 99: 357-358.

Ogata M, Hasegawa Y, Ohtani H, Mineyama M, Miura I (2008). The ZZ/ZW sex-determining mechanism originated twice and independently during evolution of the frog, Rana rugosa. Heredity 100: 92-99. 